\title{
The Concordance Rates between LV Hypertrophy and RV Hypertrophy in Patients with Hypertrophic Cardiomyopathy as Diagnosed by Cardiovascular MRI with Fibrosis Imaging
}

\author{
Nessim N. Amin*, Saundra B. Grant, June A. Yamrozik, Ronald B. Williams, \\ Diane V. Thompson, Mark Doyle, Moneal Shah, Robert W. W. Biederman \\ Gerald McGinnis Cardiovascular Institute, Allegheny General Hospital, Pittsburgh, Pennsylvania, USA \\ Email: ${ }^{\mathrm{d} r}$ nesso83@yahoo.com
}

Received 29 March 2015; accepted 21 July 2015; published 24 July 2015

Copyright (C) 2015 by authors and Scientific Research Publishing Inc.

This work is licensed under the Creative Commons Attribution International License (CC BY).

http://creativecommons.org/licenses/by/4.0/

(c) (i) Open Access

\begin{abstract}
Introduction: CMR has become the leading modality to define the clinical impact of hypertrophic cardiomyopathy (HCM). Late gadolinium enhancement (LGE) accurately identifies regions of myocardial fibrosis. It is well known that myocardial fibrosis can occur in patients with HCM and is independently linked to a poorer prognosis than those without fibrosis by CMR. Hypothesis: We hypothesize that there is significant RV involvement in HCM when incorporates a CMR analysis for RV hypertrophy and fibrosis. Methods: A retrospective review of all patients referred for HCM was performed. SSFP/LGE techniques were used to diagnose patients with HCM, using gadolinium administration (0.15 mmol/kg). Post-injection (10 minutes) LGE images were obtained using manual T1-weighted, IR-preparations. Regions of myocardium with LGE signals were visually designated as fibrotic. LV/RV mass indices (LVMI/RVMI) and ejection fractions were calculated. Results: Via 72 patients referred for HCM, 47(65\%) were CMR confirmed. The mean LVMI was $108 \pm 44 \mathrm{~g} / \mathrm{m}^{2}$ while the mean RVMI was $30 \pm 21 \mathrm{~g} / \mathrm{m}^{2}$. As well, $34 / 47(72 \%)$ had evidence of LV fibrosis while 24/47 (51\%) had evidence for RV fibrosis. Of the RVH positive patients, $26 / 34$ (76\%) patients were LV LGE positive and 18/34 (52\%) were RV LGE positive. Conclusion: The high frequency of RVH and RV fibrosis in the setting of HCM is surprising in that this phenomenon is rarely described. However, there is no reason to expect the phenotypic expression should be limited to the $\mathrm{LV}$. Interestingly, as for the LV, the presence or absence of RV fibrosis has little predictive power towards the systolic function.
\end{abstract}

\footnotetext{
"Corresponding author.

How to cite this paper: Amin, N.N., Grant, S.B., Yamrozik, J.A., Williams, R.B., Thompson, D.V., Doyle, M., Shah, M. and Biederman, R.W.W. (2015) The Concordance Rates between LV Hypertrophy and RV Hypertrophy in Patients with Hypertrophic Cardiomyopathy as Diagnosed by Cardiovascular MRI with Fibrosis Imaging. World Journal of Cardiovascular Diseases, 5, 171-180. http://dx.doi.org/10.4236/wjcd.2015.57020
} 


\section{Keywords}

\section{Cardiac MRI, Right Ventricle, Hypertrophic Cardiomyopathy, Fibrosis Imaging, Hypertrophy}

\section{Introduction}

Hypertrophic cardiomyopathy (HCM) is a genetically determined heart muscle disease most often (60 to 70 percent) caused by mutations in one of several sarcomere genes which encode components of the contractile apparatus [1]-[5]. HCM is characterized by left ventricular hypertrophy of various morphologies, with a wide array clinical manifestations and hemodynamic abnormalities [6] [7]. Infrequently, however, right ventricular (RV) involvement is described in reports of HCM. Other cardiac imaging modalities such as echocardiography and nuclear imaging have well known difficulties in assessment of the RV morphological abnormalities in HCM [8]-[10]. This has led to Cardiovascular MRI (CMR) becoming the leading modality to define the structural and functional as well as the clinical impact of HCM providing complete coverage of both ventricles with high spatial resolution Figures 1-3. Moreover, assessment of RV diseases, and specifically cardiomyopathies, is greatly

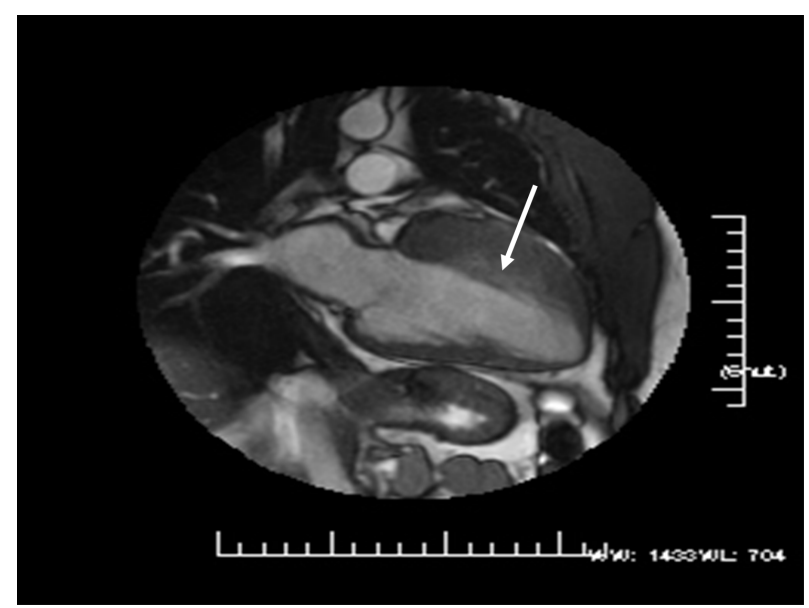

Figure 1. A 52 years old woman with SCD with CMR depicting SSFP images that demonstrate markedly thickened anterior wall to a maximum of $33 \mathrm{~mm}$.

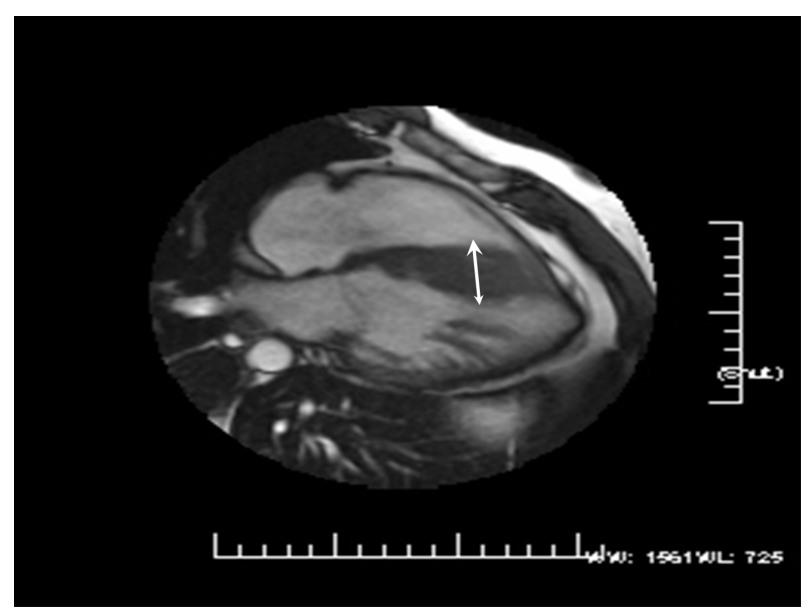

Figure 2. A 52 years old woman (same in Figure 1) with SSFP images demonstrating markedly thickened septal wall to a maximum of $37 \mathrm{~mm}$. 


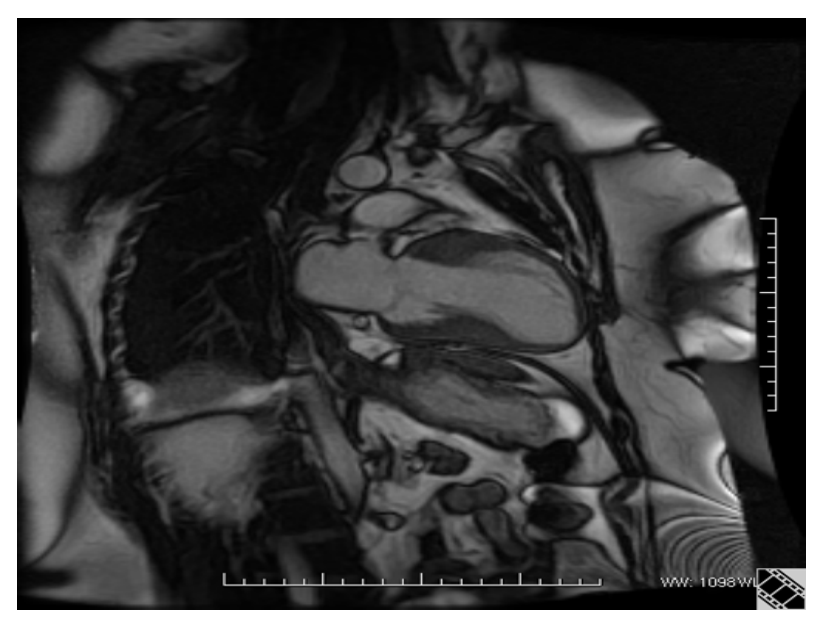

Figure 3. A 32-years old female with SSFP image depicting markedly thin apex with relative hypertrophy of basal myocardium.

aided by the use of non-contrast CMR, which can be used to measure not only the thin-walled RV, providing mass estimations, but also provide volumetric information. Late gadolinium enhancement (LGE) adds the accurate identification of regions of myocardial fibrosis Figure 4, Figure 5. Via CMR; innumerable studies have established that LVH and LGE are the predominant phenotypic expressions of HCM. We hypothesize that there is significant right ventricular myocardial fibrosis as detected by CMR in HCM patients, which upon demonstration, would provide evidence for right ventricular involvement.

\section{Methods}

Images were obtained using a 1.5T General Electric whole body scanner (HD Excite version 12 GE Milwaukee WI). Subjects were imaged in the supine position and signal reception was accomplished using a 4-channel phased array cardiac coil. Sequences of interest included single shot Echo Planar Imaging, using a cardiac-triggered system with 40mT maximum gradient strength and $150 \mathrm{mT} / \mathrm{m} / \mathrm{ms}$ maximum slew rate. The following parameters were used: repetition time $(\mathrm{TR})=9 \mathrm{~ms}$, echo time $(\mathrm{TE})=4 \mathrm{~ms}$, flip angle $(\mathrm{FA})=40$ degrees, slice thickness $=8 \mathrm{~mm}$, number of excitations $(\mathrm{NEX})=2-4$, field of view $=380-420 \mathrm{~mm}$, and matrix $128 \times 128$. Sagittal scout images were used to plan multiplanar steady state free-precession sequences (SSFP). Contrast imaging/LGE was performed with gadolinium administration ( $0.15 \mathrm{mmol} / \mathrm{kg}$, Post-injection 2/10 minutes). LGE images were obtained using breath-hold manual T1-weighted, inversion recovery preparations. Regions of myocardium with usually abnormal high signals were designated as fibrotic according to standard techniques. LV/RV mass indices (LVMI/RVMI), wall thickness and end-diastolic volumes were calculated after manually delineating the ventricular contours (epicardial and endocardial) during end-diastole. The ejection fraction was calculated by dividing LV stroke volume by end-diastolic volume. Fibrosis was semi-quantitatively assessed in both ventricles by an experienced clinician. A local IRB approved the research protocol.

\section{Statistics}

Data were reported as mean \pm standard deviation for continuous variables and percentages for categorical variables. The independent samples t-test or the Mann-Whitney U test was used to determine differences between continuous variables. The chi-square test or Fisher's Exact test was used to compare categorical variables. Data were examined for normality using the Kolmogrov-Smirnov test. Nonparametric tests were used to evaluate data that breaks parametric assumptions. A value of $\mathrm{p}<0.05$ on two-tailed testing was considered statistically significant. Statistical analyses were performed using PASW Statistics, version 18.0 (IBM-SPSS Inc., Chicago).

\section{Results}

Via 72 patients referred for HCM, presenting to Allegheny General Hospital (Pittsburgh, Pennsylvania) Cardiac 


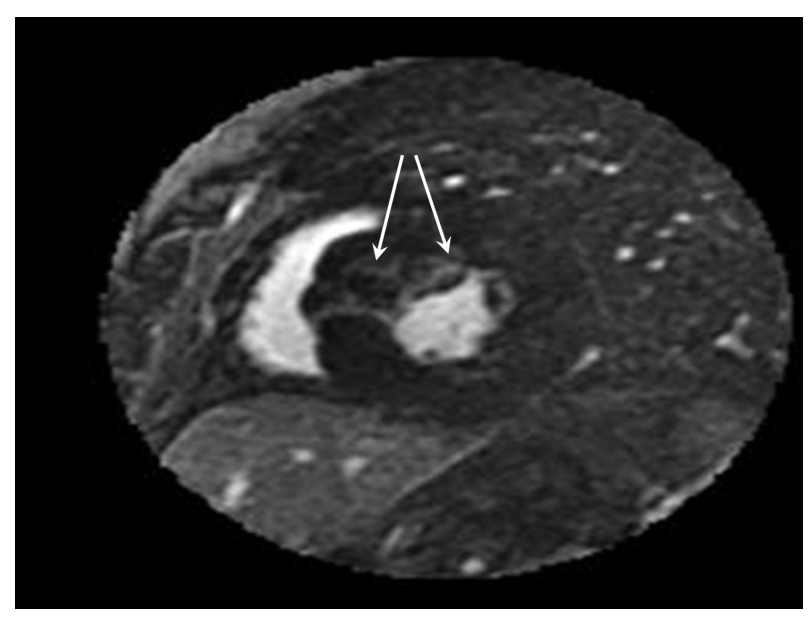

Figure 4. The same patient in Figures 1-3, late gadolinium enhancement (LGE) imaging showing the lacy pattern of fibrosis without infarct. The patient had a wall thickness $>30 \mathrm{~mm}$.

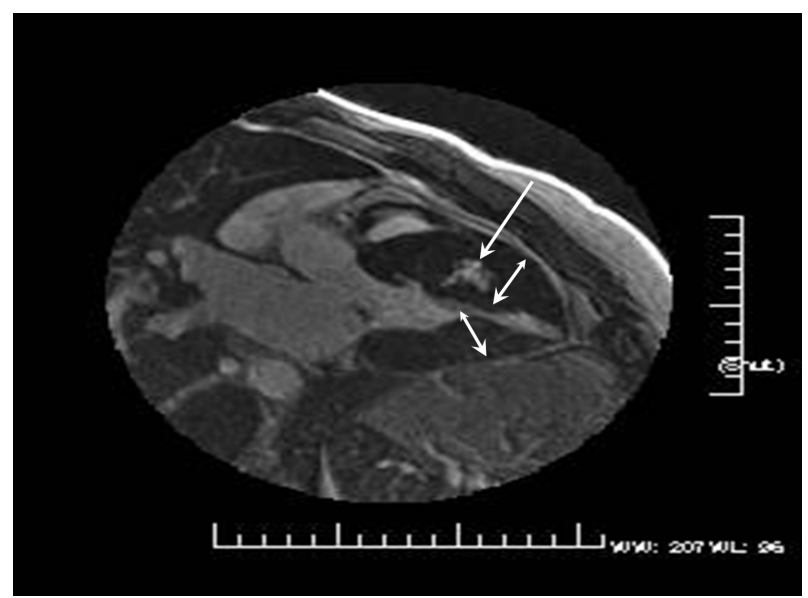

Figure 5. A 52 years old woman (same in Figure 1 \& Figure 2) with LGE images demonstrating anteroseptal hypertrophy and extensive mid septal post-gadolinium enhancement consistent with marked fibrosis and/or infarct likely outstripping the vascular supply.

MRI Center from 2007-2013, 52 (72\%) were CMR confirmed but five were excluded due to poor image quality (demographics are shown in Table 1). The mean LVMI for the remaining 47 patients was $108 \pm 44 \mathrm{~g} / \mathrm{m}^{2}$ while the mean RVMI was $30 \pm 2 \mathrm{~g} / \mathrm{m}^{2}$. All patients met formal LVH criteria while 34/47 (72\%) met RVH criteria. As well, 34/47 (72\%) had evidence of LV fibrosis while 24/47 (51\%) had evidence for RV fibrosis. Of the RVH positive patients, 26/34 (76\%) patients were LV LGE positive and over half of the patients, specifically 18/34 (52\%) were RV LGE positive. Only 2 patients with RV fibrosis had absent LV fibrosis (see Table 2). In order to ascertain whether fibrosis drove cardiac function, further parameters were performed.

Relating the presence of LGE, there was no statistical difference in LVEF\% between LV LGE positive and LV LGE negative patients ( $\mathrm{p}=\mathrm{NS}$ ). Mean LVEF\% was similar between groups (LV - DHE, Mean = 66.6, SD = 9.5, Median $=69.0$ vs. LV LGE +, Mean $=67.5, \mathrm{SD}=10.5$, Median $=69.5)$. As well, there was no statistical difference in LVEF\% between RV LGE - and RV LGE + patients ( $\mathrm{p}=\mathrm{NS}$ ). Mean LVEF\% was similar between groups $(\mathrm{RV}$ LGE - , Mean $=67.5, \mathrm{SD}=12.6$, Median $=70.0$ vs. $\mathrm{RV}$ LGE - , Mean $=67.0, \mathrm{SD}=7.4$, Median $=$ 68.0). Similarly, there was no statistical difference in RVEF\% between LV LGE - and LV LGE + patients ( $\mathrm{p}=$ NS). Mean RVEF\% was similar between groups (LV LGE - , Mean $=58.1, \mathrm{SD}=5.3$, Median $=60.0 \mathrm{vs.} \mathrm{LV}$ $\mathrm{LGE}+$, Mean $=57.6, \mathrm{SD}=8.8$, Median $=58.5$ ). 
Table 1. Demographics and cardiac MRI data for patients with HCM (N = 47).

\begin{tabular}{cc}
\hline Age (yrs) & $49 \pm 16$ \\
Men & $30 / 47$ \\
Body Surface Area $\left(\mathrm{m}^{2}\right)$ & $2 \pm 0.33$ \\
LVEDmi $\left(\mathrm{g} / \mathrm{m}^{2}\right)$ & $108 \pm 44$ \\
RVEDmi $\left(\mathrm{g} / \mathrm{m}^{2}\right)$ & $30 \pm 21$ \\
LVEDvi $\left(\mathrm{ml} / \mathrm{m}^{2}\right)$ & $80 \pm 25$ \\
RVEDvi (ml $\left./ \mathrm{m}^{2}\right)$ & $56 \pm 21$ \\
LV LGE + & $72 \%$ \\
RV LGE - & $51 \%$ \\
LVEF (\%) & $67 \pm 10$ \\
RVEF $(\%)$ & $56 \pm 8$ \\
\hline
\end{tabular}

Table 2. Demographics and cardiac MRI data for patients with RV LGE + $(\mathrm{N}=24)$ and RV LGE - $(\mathrm{N}=23)$.

\begin{tabular}{|c|c|c|}
\hline & RV LGE + & RV LGE - \\
\hline Age (yrs) & $48 \pm 16$ & $50 \pm 19$ \\
\hline Men & $18 / 24$ & $14 / 23$ \\
\hline Body Surface Area $\left(\mathrm{m}^{2}\right)$ & $2.17 \pm 0.3$ & $2.13 \pm 0.3$ \\
\hline LVEDmi (g/m²) & $126 \pm 54$ & $89 \pm 18$ \\
\hline RVEDmi (g/m²) & $33 \pm 28$ & $27 \pm 11$ \\
\hline LVEDvi (ml/m²) & $80 \pm 20$ & $80 \pm 31$ \\
\hline RVEDvi (ml/m²) & $55 \pm 22$ & $56 \pm 22$ \\
\hline LV LGE + & $22 / 24(91 \%)$ & $10 / 23(43 \%)$ \\
\hline LVEF (\%) & $65 \pm 11$ & $69 \pm 8$ \\
\hline RVEF (\%) & $56 \pm 9$ & $59 \pm 5$ \\
\hline
\end{tabular}

$\mathrm{p}=$ NS for all comparisons.

\section{Discussion}

Imaging modalities such as echocardiography and nuclear imaging help to detect various prognostic indicators in HCM such as LV mass, function and associated microvascular obstruction [11] Figure 6, Figure 7. Cardiovascular Magnetic Resonance (CMR) has become an established imaging modality which provides often unique information on a wide range of cardiovascular diseases. High resolution, absence of foreshortening and ability to perform three-dimensional (3D) imaging quickly in addition to the reliability and reproducibility has placed CMR at the forefront as the gold standard for LV/RV functional and structural assessment. The evaluation of RV is classically difficult because of its inability to conform easily to any standard geometric formulae. Assessment of RV diseases, and specifically cardiomyopathies, is greatly aided by the use of non-contrast CMR that can be used to measure not only the thin-walled RV, providing mass estimations, but also provide volumetric information. Recently, using LGE imaging, myocardial areas that demonstrate increased contrast enhancement typically correspond to areas of greatest hypertrophy, greatest myofibrillar disarray and areas of greatest collagen deposition in the exteracellular matrix Figure 8. Early evidence from several investigators has demonstrated that those presenting with a positive LGE signal within the myocardium, with specific focus on LV LGE, 


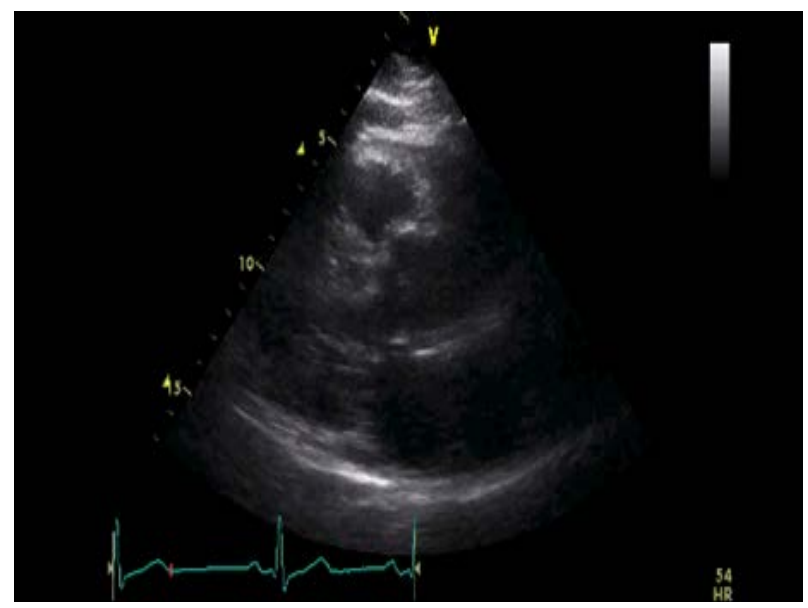

Figure 6. The same patient in Figure 3 with a parasternal long axis view that reveals the asymmetric septal hypertrophy of the left ventricle.

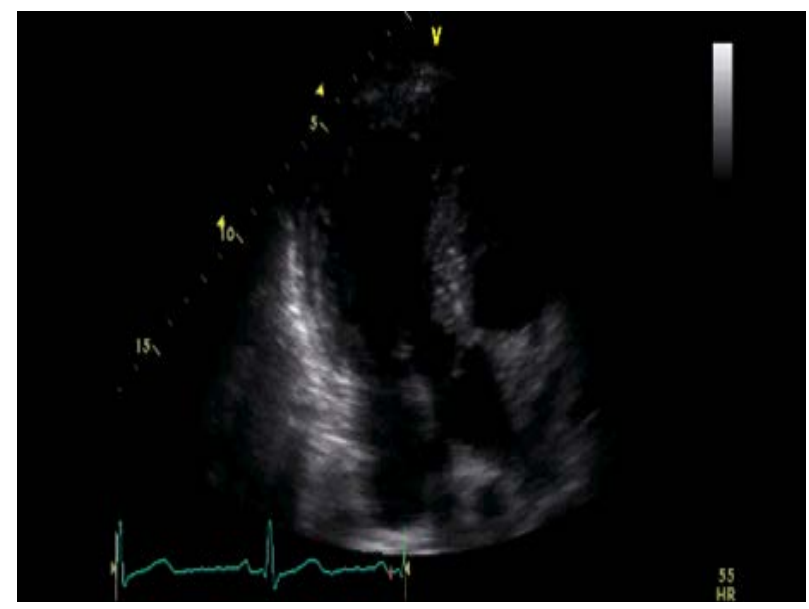

Figure 7. The same patient in Figure 3 with a 5-chamber-view via echocardiography demonstrating a similar pattern, albeit less definitive of apical thinning with relative basal hypertrophy.

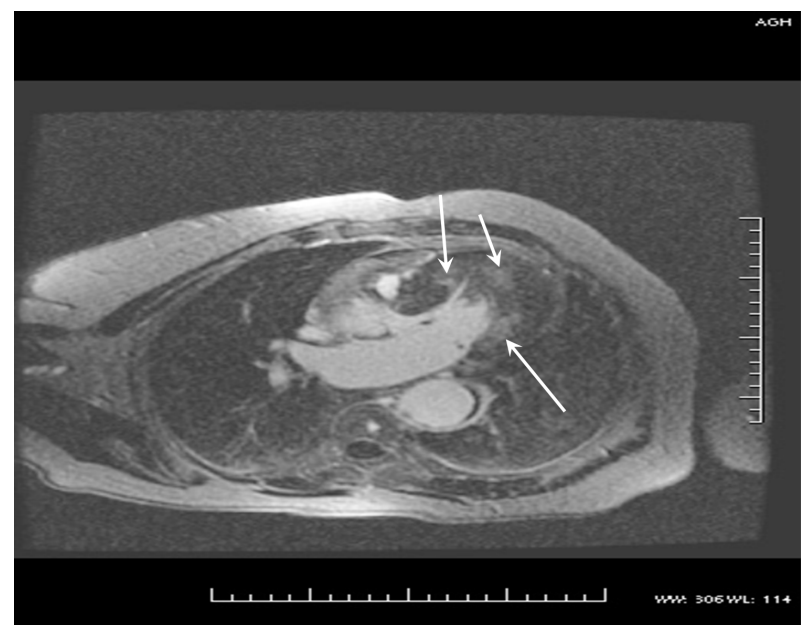

Figure 8. A 48 years old woman with documented HCM and LGE demonstrating diffuse global fibrosis indicative of gross LV fibrosis. 
possess an adverse cardiovascular outcome.

Four single-center studies focused on the use of LGE as a prognostic factor for HCM to predict ventricular tachyarrhythmia, atrial fibrillation, heart failure and sudden cardiac death demonstrated variable predictive power for VT/VF and SCD dependent on presence or absence of LGE [12]-[15]. More recently, a meta-analysis done by Green et al. incorporating these four studies in 2012 showed that there are significant predictable relationships between LGE and cardiovascular mortality, heart failure death, and all-cause mortality in HCM 16. We had performed an earlier systematic review demonstrating similar finding in which LGE, for the first time in 1064 patients did predict SCD with $\chi 2$ of 6.6 and an odds ratio of 3.3 [17]. To date, however, minimal focus has been concentrated on the RV fibrosis and LGE (in only one autopsy-based HCM report in the form of increased wall thickness) [8]. The significant high concordance rates between LV hypertrophy and RV hypertrophy in patients with HCM was previously described in an interesting study, conducted by Maron et al. in 2007, to evaluate RV wall thickness and mass indices via high spatial resolution CMR [18]. This group concluded that diffuse RV wall hypertrophy was present in high number of patients with HCM (54\%) while only one subject had CMR evidence of RV fibrosis Figures 9-11. Herein we demonstrate with high sensitivity detection, a high incidence of the RV fibrosis (52\%) in HCM patients referred to a tertiary referral center using standard LGE techniques. Our results provide support for and emphasize the hypothesis that the genetic cardiomyopathic pathology

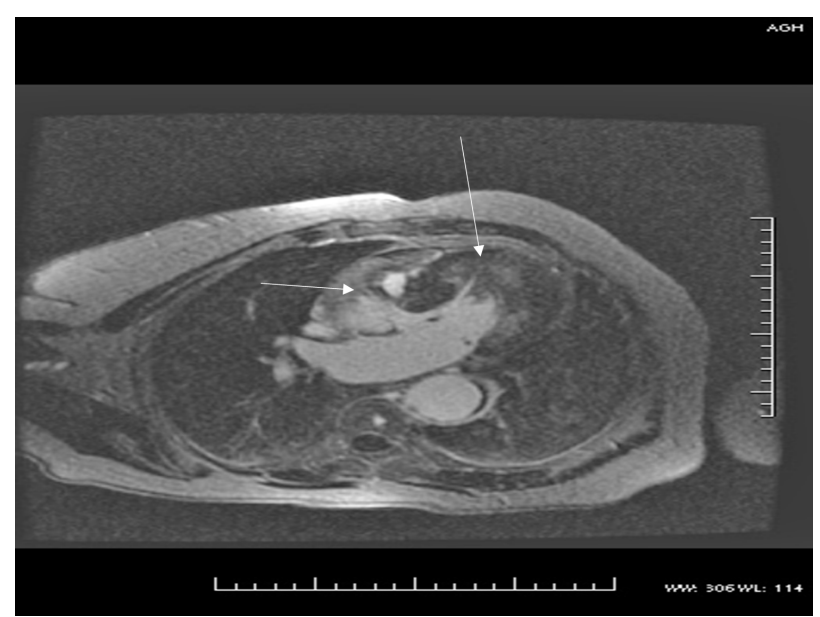

Figure 9. Late gadolinium enhancement (LGE) demonstrating diffuse enhancement of the LV and RV. Note, more RV outflow tract LGE is present than LV LGE in a proven HCM patient.

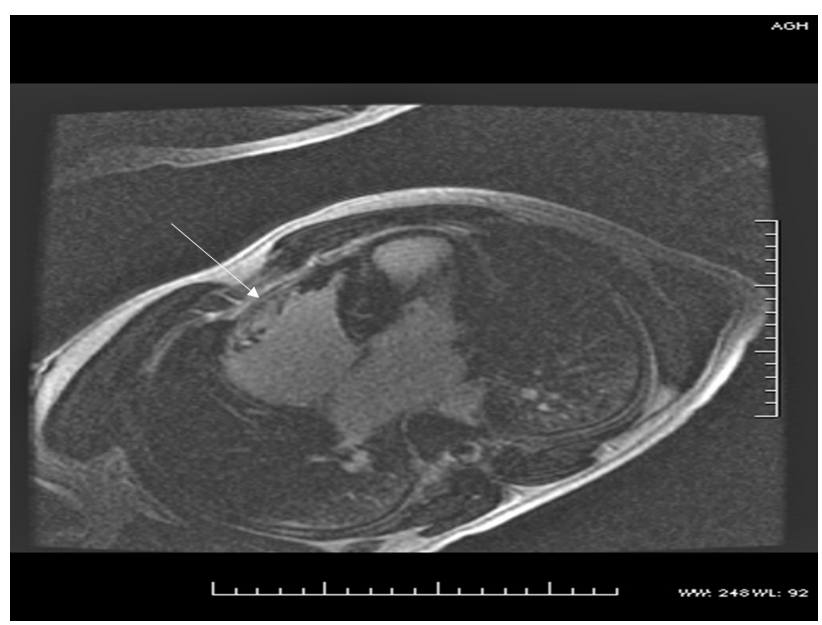

Figure 10. LGE of both ventricles is more evident in a near transmural but irregular RV free wall enhancement pattern from a young man with a father as the HCM proband. 


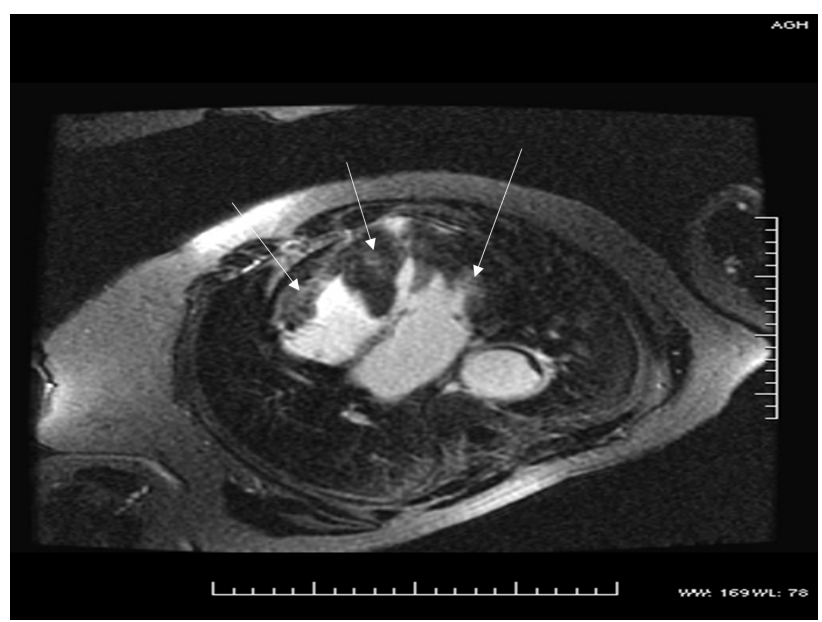

Figure 11. LGE showing irregular, diffuse biventricular enhancement indicative of diffuse fibrosis consistent with the clinical history of HCM.

in HCM is more diffuse involving a biventricular process (our study focused on the myocardial fibrotic changes) and bring attention to reconsidering the AHA definition for the HCM that focuses only on the LV pathology [19].

The frequency of RVH and RV fibrosis in the setting of HCM is surprising in that this phenomenon is rarely described [20]-[22]. However, given the genetic abnormalities, there is no reason to expect that the phenotypic expression should be limited to the LV. Interestingly, as for the LV, the presence or absence of RV fibrosis had little predictive power towards the systolic function based on our study results. Finally, our study raises a question: "Do HCM patients with evidence of combined RV and LV myocardial fibrosis have worse outcomes or higher mortality rates compared to HCM patients with LV or RV fibrosis alone?” We believe that further prospective clinical studies are needed to answer this question.

\section{Conclusion}

Classically, HCM has been assumed to be purely LV pathology. Via CMR, over half of tertiary referral population was shown via LGE fibrosis imaging to have not only RVH but RV fibrosis. This finding provides further insight into the biventricular nature of HCM pathophysiology and raises further questions as an additional adverse prognostic factor.

\section{Acknowledgements}

Participated in the research design: Biederman, Grant. Participated in writing of the manuscript: Amin, Biederman. Participated in the performance of the research: Amin, Grant, Biederman, Shah. Participated in data analysis: Thompson, Doyle, Biederman. Contributed new analytic tools: Yamrozik, Williams, Grant.

\section{Disclosures}

All authors listed have no conflicts of interest to disclose and none of the authors have received any funding from any resource.

\section{References}

[1] Maron, B.J. (2002) Hypertrophic Cardiomyopathy: A Systematic Review. JAMA, 287, 1308-1320. http://dx.doi.org/10.1001/jama.287.10.1308

[2] Maron, B.J., McKenna, W.J., Danielson, G.K., et al. (2003) American College of Cardiology/European Society of Cardiology Clinical Expert Consensus Document on Hypertrophic Cardiomyopathy: A Report of the American College of Cardiology Foundation Task Force on Clinical Expert Consensus Documents and the European Society of Cardiology Committee for Practice Guidelines. Journal of the American College of Cardiology, 42, 1687-1713. 
http://dx.doi.org/10.1016/S0735-1097(03)00941-0

[3] Elliott, P.M., Poloniecki, J., Dickie, S., et al. (2000) Sudden Death in Hypertrophic Cardiomyopathy: Identification of High Risk Patients. Journal of the American College of Cardiology, 36, 2212-2218. http://dx.doi.org/10.1016/S0735-1097(00)01003-2

[4] Spirito, P., Bellone, P., Harris, K.M., Bernabo, P., Bruzzi, P. and Maron, B.J. (2000) Magnitude of Left Ventricular Hypertrophy and Risk of Sudden Death in Hypertrophic Cardiomyopathy. The New England Journal of Medicine, 342, 1778-1785. http://dx.doi.org/10.1056/NEJM200006153422403

[5] Wigle, E.D., Rakowski, H., Kimball, B.P. and Williams, W.G. (1995) Hypertrophic Cardiomyopathy: Clinical Spectrum and Treatment. Circulation, 92, 1680-1692. http://dx.doi.org/10.1161/01.CIR.92.7.1680

[6] Seidman, J.G. and Seidman, C. (2001) The Genetic Basis for Cardiomyopathy: From Mutation Identification to Mechanistic Paradigms. Cell, 104, 557-567. http://dx.doi.org/10.1016/S0092-8674(01)00242-2

[7] Richard, P., Charron, P., Carrier, L., et al. (2003) EUROGENE Heart Failure Project. Hypertrophic Cardiomyopathy: Distribution of Disease Genes, Spectrum of Mutations, and Implications for a Molecular Diagnosis Strategy. Circulation, 107, 2227-2232. http://dx.doi.org/10.1161/01.CIR.0000066323.15244.54

[8] Mozaffarian, D. and Caldwell, J.H. (2001) Right Ventricular Involvement in Hypertrophic Cardiomyopathy: A Case Report and Literature Review. Clinical Cardiology, 24, 2-8. http://dx.doi.org/10.1002/clc.4960240102

[9] McKenna, W.J., Kleinebenne, A., Nihoyannopoulos, P. and Foale, R. (1988) Echocardiographic Measurements of Right Ventricular Wall Thickness in Hypertrophic Cardiomyopathy: Relation to Clinical and Prognostic Features. Journal of the American College of Cardiology, 11, 351-358. http://dx.doi.org/10.1016/0735-1097(88)90101-5

[10] Severino, S., Caso, P., Cicala, S., et al. (2001) Involvement in Right Ventricle in Left Ventricular Hypertrophy: Analysis by Pulsed Doppler Tissue Imaging. European Journal of Echocardiography, 1, 281-288.

http://dx.doi.org/10.1053/euje.2000.0043

[11] Noureldin, R.A., Liu, S., Nacif, M.S., et al. (2012) The Diagnosis of Hypertrophic Cardiomyopathy by Cardiovascular Magnetic Resonance. Journal of Cardiovascular Magnetic Resonance, 14, 17. http://dx.doi.org/10.1186/1532-429X-14-17

[12] Papavassiliu, T., Germans, T., Flüchter, S., et al. (2009) CMR Findings in Patients with Hypertrophic Cardiomyopathy and Atrial Fibrillation. JCMR, 11, 34.

[13] Petkow-Dimitrow, P., Klimeczek, P., Vliegenthart, R., Pasowicz, M., Miszalski-Jamka, T., Oudkerk, M., Podolec, P., Dubiel, J.S. and Tracz, W. (2009) Late Gadolinium Enhancement in Cardiovascular Magnetic Resonance in Patients with Hypertrophic Cardiomyopathy Complicated by Life-Threatening Ventricular Tachyarrhythmia. Kardiologia Polska, 67, 964-969.

[14] Maron, M.S. (2012) Clinical Utility of Cardiovascular Magnetic Resonance in Hypertrophic Cardiomyopathy. Journal of Cardiovascular Magnetic Resonance, 14, 13.

[15] Alla, V.M., Koneru, S., Hunter, C. and Mooss, A. (2012) LGE and the Risk of Sudden Death in HCM. JACC: Cardiovascular Imaging, 5, 761-762. http://dx.doi.org/10.1016/j.jcmg.2012.05.004

[16] Green, J.J., Berger, J.S., Kramer, C.M. and Salerno, M. (2012) Prognostic Value of Late Gadolinium Enhancement in Clinical Outcomes for Hypertrophic Cardimyopathy. JACC: Cardiovascular Imaging, 5, 370-377. http://dx.doi.org/10.1016/j.jcmg.2011.11.021

[17] Silva, N., Paladino, A., Doyle, M., Reddy, S.T., et al. (2012) A Systematic Review for Sudden Cardiac Death in Hypertrophic Cardiomyopathy Patients with Myocardial Fibrosis: A CMR LGE Study. Circulation, 124, Article ID: A15932.

[18] Maron, M.S., Hauser, T.H., Dubrow, E., et al. (2007) Right Ventricular Involvement in Hypertrophic Cardiomyopathy. American Journal of Cardiology, 100, 1293-1298.

[19] Maron, B.J., Towbin, J.A., Thiene, G., et al. (2006) American Heart Association; Council on Clinical Cardiology, Heart Failure and Transplantation Committee; Quality of Care and Outcomes Research and Functional Genomics and Translational Biology Interdisciplinary Working Groups; Council on Epidemiology and Prevention. Contemporary definitions and classification of the cardiomyopathies: An American Heart Association scientific statement from the Council on Clinical Cardiology, Heart Failure and Transplantation Committee; Quality of Care and Outcomes Research and Functional Genomics and Translational Biology Interdisciplinary Working Groups; and Council on Epidemiology and Prevention. Circulation, 113, 1807-1816. http://dx.doi.org/10.1161/CIRCULATIONAHA.106.174287

[20] Chan, R.H., Maron, B.J., Olivotto, I., et al. (2015) Significance of Late Gadolinium Enhancement at Right Ventricular Attachment to Ventricular Septum in Patients with Hypertrophic Cardiomyopathy. American Journal of Cardiology, 116, 436-441.

[21] Zhang, S., Yang, Z.G., Sun, J.Y., et al. (2014) Assessing Right Ventricular Function in Patients with Hypertrophic Cardiomyopathy with Cardiac MRI: Correlation with the New York Heart Function Assessment (NYHA) Classifica- 
N. N. Amin et al.

tion. PLOS ONE, 9, e104312.

[22] Bravo, P.E., Luo, H.C., Pozios, I., et al. (2015) Late Gadolinium Enhancement Confined to the Right Ventricular Insertion Points in Hypertrophic Cardiomyopathy: An Intermediate Stage Phenotype? European Heart Journal-Cardiovascular Imaging (In Press). http://dx.doi.org/10.1093/ehjci/jev154 